\title{
The Existence of an Old Quasar at $z=3.91$ and its Implications for $\Lambda(t)$ Deflationary Cosmologies
}

\author{
J. V. Cunha* and R. C. Santos ${ }^{\dagger}$ \\ Departamento de Física, \\ Universidade Federal do Rio Grande do Norte, C.P. 1641, 59072-970, Natal, RN, Brasil
}

(November 1, 2018)

\begin{abstract}
We investigate some observational constraints on decaying vacuum cosmologies based on the recently discovered old high redshift quasar APM $08279+5255$. This object is located at $z=3.91$ and has an estimated age of 2-3 Gyr. The class of $\Lambda(t)$ cosmologies is characterized by a positive $\beta$ parameter smaller than unity which quantifies the ratio between the vacuum and the total energy density. Assuming the lower limit age (2 Gyr) and that the cold dark matter contributes with $\Omega_{\mathrm{M}}=0.2$ we show that $\beta$ is constrained to be $\geq 0.07$ while for an age of 3 Gyr and $\Omega_{\mathrm{M}}=0.4$ the $\beta$ parameter must be greater than 0.32 . Our analysis includes closed, flat and hyperbolic scenarios, and it strongly suggests that there is no age crisis for this kind of $\Lambda(t)$ cosmologies. Lower limits to the redshift quasar formation are also briefly discussed to the flat case. For $\Omega_{\mathrm{M}}=0.4$ we found that the redshift formation is constrained by $z_{f} \geq 8.0$.
\end{abstract}

\footnotetext{
*jvital@dfte.ufrn.br

${ }^{\dagger}$ rose@dfte.ufrn.br
} 


\section{INTRODUCTION}

Recent observations from Supernovae (SNe) type Ia strongly suggest that the bulk of energy in the Universe is repulsive and appears like a dark component; an unknown form of energy with negative pressure [in addition to the ordinary dark matter] which is probably of primordial origin [1]. The most natural candidate for dark energy is the cosmological constant $(\Lambda)$, or equivalently, a perfect fluid obeying the equation of state, $p_{v}=-\rho_{v}$, which is usually interpreted as the constant vacuum energy density of all fields existing in the Universe. The $\Lambda$-term is the simplest but not the unique possibility. Other candidates appearing in the literature are: a relic scalar field component (SFC) which is slowly rolling down its potencial [2], a decaying vacuum energy density, or a time varying $\Lambda$-term [3], the so-called "X-matter", an extra component [4] characterized by an equation of state $p_{\mathrm{x}}=\omega \rho_{\mathrm{x}}$ (XCDM), and the Chaplygin type gas whose equation of state is $p=-A / \rho^{\alpha}$, where $A$ and $\alpha$ are positive constants [5] (see also Lima [6] for a quick review). On the other hand, the existence of old high-redshift objects is one of the best methods for constraining the age of the Universe, as well the basic cosmological parameters [7]. Such objects also provide an important key for determining the first epoch of galaxy formation. In this connection, quasars are among the most luminous objects known in the universe and their prominent emission-lines contains valuable information to estimate their ages. The recently reported age estimates of the APM 08279+5255 quasar with a lower limit of 2-Gyr-old at redshift $z=3.91$ is therefore a particularly interesting event [8]. In this article we investigate some cosmological implications from the existence of this quasar to a large class of decaying vacuum cosmologies proposed by Lima and collaborators [9]. Some constraints on the first epoch of quasar formation are also discussed. 


\section{OLD HIGH-Z OBJECTS AND AGE-REDSHIFT TEST}

Several groups have searched for old objects at high redshifts [10]. Such discoveries accentuated even further the already classical "age crisis" and gave rise to a new variant of this problem, which could be named the high-z time scale crisis [11]. More recently, Hasinger and co-workers [8] reported the discovery of an ionized Fe K edge in the $z=3.91$ broad absorption line quasar APM 08279+5255. The Fe/O ratio of the absorption material is significantly higher than solar abundance $(\mathrm{Fe} / \mathrm{O}=2-5)$, and requires a timescale of $\sim 3$ Gyr with a conservative lower limit age of 2 Gyr. The Einstein-de Sitter model is not able to explains the age of this object $[8,12]$. Let us now examine how the existence of this quasar constrains the basic parameters defining the time varying $\Lambda(t)$ cosmologies. The general age-redshift relation in the $\Lambda(t)$ models proposed by Lima and coworkers [9] is

$$
t_{z}=H_{o}^{-1} \int_{0}^{(1+z)^{-1}} \frac{d x}{\left[1-\frac{\Omega_{\mathrm{M}}}{(1-\beta)}+\frac{\Omega_{\mathrm{M}}}{(1-\beta)} x^{3 \beta-1}\right]^{\frac{1}{2}}} \equiv H_{o}^{-1} f\left(\Omega_{\mathrm{M}}, \beta, z\right) .
$$

For $\beta=0$ and $\Omega_{M}=1.0$ the above expression reduces to $t_{z}=\frac{2}{3} H_{o}^{-1}(1+z)^{-3 / 2}$, which is the prediction of the Einstein-de Sitter model [13]. In this case, the age of the Universe at redshift 3.91 is nearly 1 Gyr which is much less than the lower limit of the quasar age. Following standard lines, we take for granted that the age of the Universe at the specific redshift is bigger than or at least equal to the age of the quasar, namely:

$$
\frac{t_{z}}{t_{q}}=\frac{f\left(\Omega_{\mathrm{M}}, \beta, z\right)}{H_{o} t_{q}} \geq 1
$$

where $t_{q}$ is the estimated age of the quasar and $f\left(\Omega_{\mathrm{M}}, \beta, z\right)$ is the dimensionless factor defined by Eq. (1). Note that the denominator of the above expression defines a dimensionless age parameter $T_{q}=H_{o} t_{q}$. For the lower limit age of the quasar its value and the most recent determinations of the Hubble parameter $\left(H_{o}=72 \pm 8 \mathrm{kms}^{-1} \mathrm{Mpc}^{-1}\right)$ [14], such a quantity takes values on the interval $0.131 \leq T_{q} \leq 0.163$ so that $T_{q} \geq 0.131$. Therefore, for a given value of $H_{o}$, only models having an expanding age bigger than this value at $z=3.91$ will be compatible with the existence of this object. In order to assure the robustness of our 
analysis, we will adopt in our computations the lower bound for the above mentioned value of the Hubble parameter, i.e., $H_{o}=64 \mathrm{kms}^{-1} \mathrm{Mpc}^{-1}$.

The figures above show the dimensionless age parameter $T_{z}=H_{o} t_{z}$ (see Eq. (1)) as a function of the redshift for several values of $\beta$ with $\Omega_{\mathrm{M}}=0.2(\mathrm{a}, \mathrm{b})$, and $\Omega_{\mathrm{M}}=0.4$ (c,d). The shadowed regions in the graphs were determined from the minimal value of $T_{q}$. Models with curves crossing the rectangles are ruled out because they yield an age parameter smaller than the minimal value required by the presence of the quasar APM $08279+5255$. By assuming an age estimate of 2 Gyr one may see from plot (a) that the minimal value for the vacuum energy density is $\beta \geq 0.07$, which provides a minimal total age of $\sim 13.4$ Gyr. From Figure (b), we see that the minimal value of $\beta$ required is 0.31 . For $\Omega_{\mathrm{M}}=0.4$, the minimal values of $\beta$ are 0.15 and 0.32 for 2 and 3 Gyr, respectively (see also table arising on Figure 2). Such limits are less stringents than the values inferred from angular size-redshift

relation where $\beta$ falls on the interval [15] $[0.58,0.76]$. However, we stress that constraints from angular size redshift relation should be taken with some caution because a statistical analysis describing the intrinsic length distribution of the sources is still lacking. Further, although being relatively easy for decaying vacuum cosmologies to solve the age-redshift problem, the same does not happens for other classes of dark energy models like constant $\Lambda$ or XCDM cosmologies $[11,12]$.

\section{IMPLICATIONS ON THE EPOCH OF QUASAR FORMATION}

In the present section, we discuss how the age estimates of the quasar APM $08279+5255$ constrain the epoch of quasar formation. Since we have neglected a possible incubation time in our computations, the obtained redshift formation $z_{f}$ will be a conservative lower bound. For look-back time calculations, such a hypothesis can be translated as [11]

$$
t_{o b s}-t_{z_{f}}=H_{o}^{-1} \int_{\frac{1}{1+z_{f}}}^{\frac{1}{1+z_{o b s}}} \frac{d x}{\left[1-\frac{\Omega_{\mathrm{M}}}{(1-\beta)}+\frac{\Omega_{\mathrm{M}}}{(1-\beta)} x^{3 \beta-1}\right]^{\frac{1}{2}}} \geq t_{q}
$$


where the inequality signal comes from the fact that the Universe is older than or at least has the same age of any observed structure. Naturally, models for which $z_{f} \rightarrow \infty$ are clearly incompatible with the existence of this particular object, being ruled out in a natural way. In Fig. 2 we show the $z_{f}-\Omega_{\mathrm{M}}$ plane allowed by the existence of the quasar APM $08279+5255$ for $\Lambda(t)$ models. As before, two age estimates were assumed: 2 Gyr (solid curve) and 3 Gyr (dashed curve). As should be physically expected, since the effect of dark matter is decelerate the cosmic expansion ${ }^{1}$, the larger the contribution of $\Omega_{\mathrm{M}}$ the larger the value of $z_{f}$ that is required in order to account for the existence of this quasar within these cosmological scenarios.

In this way, the smallest value for the formation redshift occurs for a completely empty universe $\left(\Omega_{\mathrm{M}}=0\right)$. We find $z_{f} \geq 5$. For a low-density universe with $\Omega_{\mathrm{M}}=0.2$ we obtain $z_{f} \geq 5.85$. For an universe with $\Omega_{\mathrm{M}}=0.4$ we obtain $z_{f} \geq 8.04$. The basic results are presented in the Table appearing in Figure 2.

Summarizing, we have investigated some observational constraints on deflationary cosmologies provided by the age of the old quasar APM 08279+5255. If the estimated age of this object is correct, at light of the above results, we conclude that such models are more efficient to explain the old quasar at $z=3.91$ than constant $\Lambda$ dark energy models. Even for a value of $\beta \geq 0.07$ the age crisis at high $z$ is solved. Concerning the first epoch of quasar formation the minimal redshift formation required is $z_{f} \geq 8.0$. Naturally, new tests and analysis must be developed in order to know if deflationary cosmologies may be realistic models of the Universe.

Acknowledgements:The authors are grateful to Prof. J.A.S. Lima for helpful discussions. This work was supported by CAPES (Brazilian Research Agency).

\footnotetext{
${ }^{1}$ It means that the look-back time between the observed redshift $z_{o b s}$ and $z_{f}$ is smaller for larger values of $\Omega_{\mathrm{M}}$.
} 


\section{REFERENCES}

[1] S. Perlmutter et al., Nature 391, 51 (1998); A. G. Riess et al., AJ 116, 1009 (1998).

[2] R. R. Caldwell, Braz. J. Phys. 30, 215 (2000); P. J. E. Peebles and B. Ratra, Rev. Mod. Phys. 75, 559 (2003).

[3] M. Ozer and M. O. Taha, Phys. Lett. B171, 363 (1986); Nucl. Phys. B287, 776 (1987); K. Freese et al., Nucl. Phys. B287, 797 (1987); W. Chen and Y-S. Wu, Phys. Rev. D41, 695 (1990); J. C. Carvalho, J. A. S. Lima and I. Waga, Phys. Rev. D46 2404 (1992); F. M. Overduin and F. I. Cooperstock, Phys. Rev. D58, 043506 (1998).

[4] M. S. Turner and M. White, Phys. Rev. D56, R4439 (1997); T. Chiba, N. Sugiyama, N. and T. Nakamura, MNRAS 289, L5 (1997).

[5] M. C. Bento, O. Bertolami and A. A. Sen, Phys. Rev. D66, 043507 (2002).

[6] J. A. S. Lima, "Alternative Dark Energy Models: An Overview", astro-ph/0402109.

[7] L. Krauss, ApJ 480, 466 (1997); J. S. Alcaniz and J. A. S. Lima, ApJ 521, L87 (1999); ApJ 550, L133 (2001).

[8] S. Komossa and G. Hasinger, in XEUS studying the evolution of the universe, eds. G. Hasinger et. al (MPE Report, 2003), p. 281; G. Hasinger, N. Schartel and S. Komossa, ApJ 573, L77 (2002).

[9] J. A. S. Lima and J. M. F. Maia, Phys. Rev D49, 5597 (1994); J. A. S. Lima and M. Trodden, Phys. Rev. D53, 4280 (1996); J. V. Cunha, J. A. S. Lima and N. Pires, A \& A 390, 809 (2002).

[10] J. S. Dunlop et al., Nature 381, 581 (1996); H. Spinrard et al., ApJ 484, 581 (1997); Y. Yoshii, T. Tsujimoto and K. Kawara, ApJ 507, L133 (1998); J. S. Dunlop, in The Most Distant Radio Galaxies, ed. H. J. A. Rottgering, P. Best, and M. D. Lehnert, Dordrecht (Kluwer, Amsterdam, 1999), p. 71; L. A. Nolan, J. S. Dunlop and R. Jimenez, MNRAS 
323, 385 (2001).

[11] J. A. S. Lima and J. S. Alcaniz, MNRAS 317, 893 (2000).

[12] J. S. Alcaniz, J. A. S. Lima, and J. V. Cunha, MNRAS 340, L39 (2003).

[13] E. W. Kolb, M. S. Turner, in The Early Universe, Addison-Wesley, Redwood City (1990).

[14] W. Freedman et al., ApJ 553, 47 (2001).

[15] J. V. Cunha, J. S. Alcaniz, J. S. and J. A. S. Lima Phys. Rev. D66, 023520 (2002). 


\section{FIGURES}

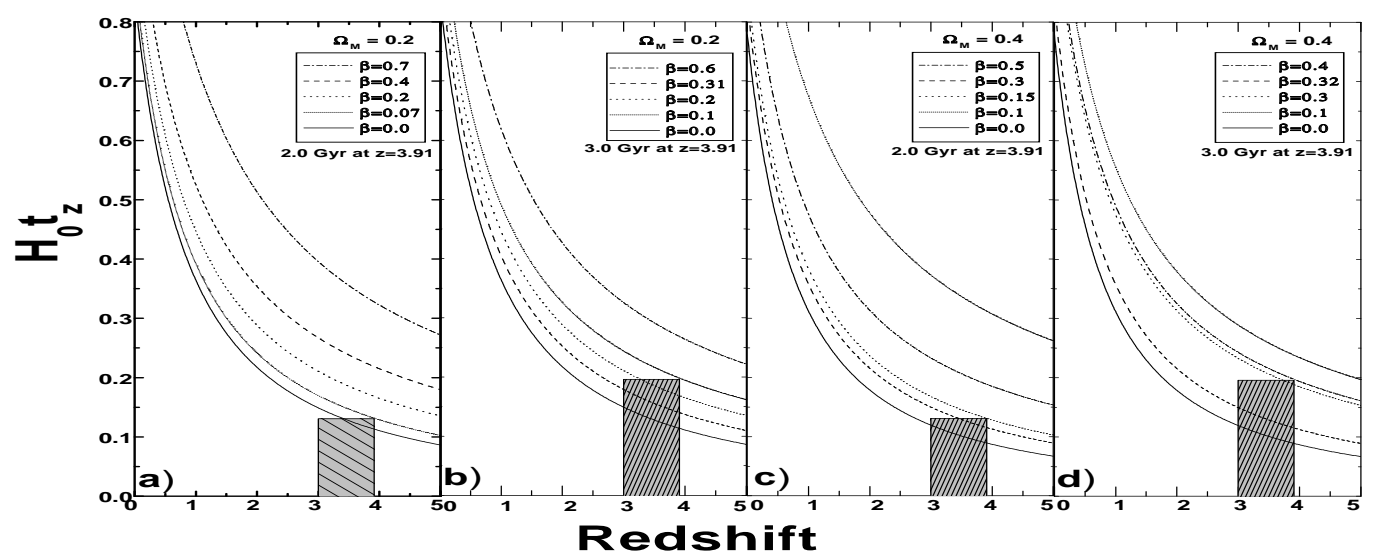

FIG. 1. Dimensionless age parameter as a function of redshift for some values of $\beta$ with $\Omega_{\mathrm{M}}=0.2$ and $\Omega_{\mathrm{M}}=0.4$. As explained in the text, all curves crossing the shadowed area yield an age parameter smaller than the minimal value required by the quasar APM $08279+5255$. Note that figures (b) and (d) are the same as in Panel (a) and (c), but for an estimate age of 3 Gyr.

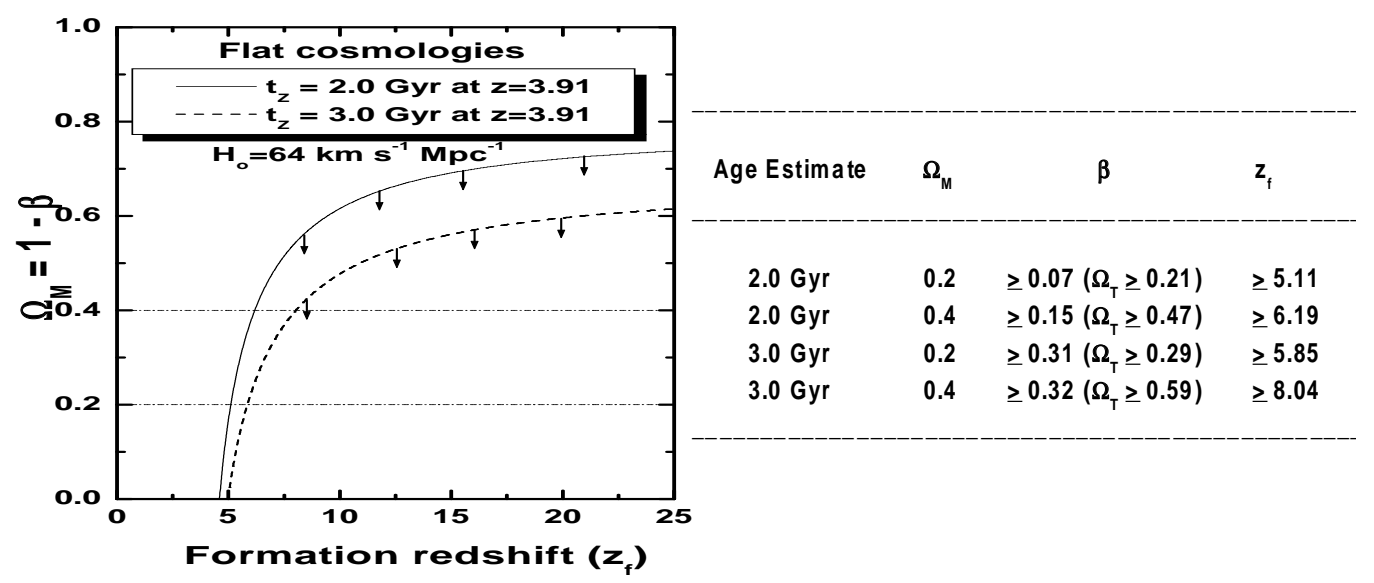

FIG. 2. The formation redshift versus $\Omega_{\mathrm{M}}$ plane for flat $\Lambda(t)$ models. Countours of a fixed age parameter $H_{o} t_{z}$ for the quasar APM $08279+5255$. The solid curve corresponds to an age estimate of 2 Gyr while the dashed one stands for 3 Gyr. For each contour the arrows point to the allowed parameter space. 